\title{
Revisão integrativa sobre a violência por parceiro íntimo na gestação
}

\author{
Integrative review regarding intimate partner violence in pregnancy
}

Revisión integrativa sobre violencia por compañero íntimo en la gestación

Maria Inês Rosselli Puccia ${ }^{1}$, Marli Villela Mamede²

\section{RESUMO}

A violência por parceiro íntimo (VPI) representa uma violação de direitos humanos e um problema de saúde pública que predominantemente afeta mulheres na idade reprodutiva. Esta revisão integrativa analisou a prevalência e fatores associados à VPI durante o período da gestação, a partir de 45 produções científicas das bases das Ciências da Saúde em Geral, durante os anos de 2007 e 2012. A prevalência de VPI na gestação atual variou entre $3 \%$ e $43 \%$, de acordo com o tipo de violência investigada e métodos de estudo. A VPI está associada a efeitos adversos à saúde materna, tais como estresse, ansiedade e depressão, tabagismo, abuso de álcool, gravidez indesejada, aborto, descolamento prematuro de placenta, préeclampsia, início tardio do pré-natal, entre outros. Conclui-se que se faz importante a investigação de VPI no pré-natal e considera-se que enfermeiros ocupam posição privilegiada para rastrear VPI na gestação e incluir as mulheres maltratadas em serviços de apoio social e comunitário.

Descritores: Violência contra a Mulher; Violência Doméstica; Mulheres Maltratadas; Violência; Gravidez.

\begin{abstract}
Intimate partner violence (IPV) represents a violation of human rights and a public health problem that increasingly affects women of reproductive age. This integrative review analyzed the prevalence and factors associated with IPV during the gestational period, including 45 scientific productions from the databases of Health Sciences in General, spanning the years 2007 to 2012. The prevalence of IPV in pregnancy varied between $3 \%$ and $43 \%$, according to the studied type of violence and study methods. IPV is associated with adverse effects to the mother's health, such as stress, anxiety and depression, tobacco use, alcohol abuse, undesired pregnancy, abortion, abruptio placentae, preeclampsia and a late start to prenatal care, among others. The authors conclude that it is important to study IPV in the prenatal period and consider that nurses occupy a privileged position to monitor IPV during pregnancy and should include mistreated women in social and community support services.
\end{abstract}

Descriptors: Violence Against Women; Domestic Violence; Battered Women; Violence; Pregnancy.

\section{RESUMEN}

La violencia por compañero íntimo (VPI) representa una violación de derechos humanos, es un problema de salud pública que afecta particularmente a mujeres en edad reproductiva. Esta revisión integrativa analizó la prevalencia y factores asociados a la VPI durante el período gestacional, partiendo de 45 producciones científicas de las bases de Ciencias de Salud General, durante 2007 y 2012. La prevalencia de VPI gestacional actual varió entre 3\% y 43\%, según el tipo de violencia investigada y métodos de estudio. Está asociada a efectos adversos a la salud materna como estrés, ansiedad y depresión, tabaquismo, alcoholismo, embarazo indeseado, aborto, desprendimiento prematuro de la placenta, preeclampsia, inicio tardío del prenatal, entre otros. Se concluye en la importancia de la investigación de VPI en el prenatal y se considera que los enfermeros ostentan un lugar privilegiado para detectar VPI gestacional e incluir a las mujeres maltratadas en servicios de apoyo social comunitario.

Descriptores: Violencia contra la Mujer; Violencia Doméstica; Mujeres Maltratadas; Violencia; Embarazo.

\footnotetext{
${ }^{1}$ Enfermeira, Mestre em Saúde Pública. Discente do Programa Interunidades de Doutoramento em Enfermagem da Universidade de São Paulo (USP). Ribeirão Preto, SP, Brasil. E-mail: puccia@usp.br.

2 Enfermeira, Doutora em Enfermagem. Professora Titular da Escola de Enfermagem de Ribeirão Preto da USP. Ribeirão Preto, SP, Brasil. E-mail: mavima@eerp.usp.br.
} 


\section{INTRODUÇÃO}

A violência por parceiros íntimos (VPI) é reconhecida como uma violação dos direitos universais e, embora represente um fenômeno da vida privada, configura-se em um importante problema de saúde pública. Neste sentido, apresenta-se como um novo tema a ser incluído no campo da atenção à saúde, com repercussões significativas na qualidade e na humanização da assistência.

Embora os dados de prevalência sejam diversos, os estudos disponíveis mostram a alta magnitude da VPI e associação com vários efeitos adversos sobre a saúde das gestantes e dos recém-nascidos ${ }^{(1)}$.

Entretanto, as repercussões da violência doméstica sobre a saúde das mulheres, que particularmente podem ser agredidas ou maltratadas durante a gravidez ou no período pós-parto, invariavelmente não são identificadas como tal. Entende-se que isto ocorra porque na prática assistencial em saúde não se fala e não se pergunta sobre violência contra mulher(2). A VPI insere-se no campo da violência de gênero e representa uma questão da vida íntima, de difícil abordagem pelo modelo clássico da clínica que é baseado na queixa-conduta. Ademais, os profissionais da saúde, de uma maneira geral, não estão preparados para inquirir as mulheres sobre temáticas sensíveis e que em tese, se enquadrem no campo psicossocial(3).

Acrescenta-se que os roteiros, formulários e fichas de atendimento, também não estão formatados para incluir tais questionamentos que, em geral, ampliam o diálogo para além da queixa que motivou a cliente a procurar por atendimento.

Trata-se, portanto, de uma questão de invisibilidade do problema no campo da atenção à saúde que requer, primeiramente, a apreensão de sua magnitude para que, posteriormente, seja incentivada a adoção de medidas que corroborem com a incorporação de métodos de rastreamento, mediante o aprimoramento das equipes de saúde.

Considerando-se a relevância deste tema, o presente estudo tem por objetivo analisar, por meio de uma revisão integrativa da produção científica, a magnitude da VPI na gestação, destacando-se a prevalência e as consequências deste agravo sobre a saúde materna.

\section{MATERIAIS E MÉTODOS}

Trata-se de uma revisão integrativa dos últimos cinco anos, relacionada à VPI vivenciada por gestantes. De acordo com o objetivo do estudo, optou-se por este tipo de revisão de literatura, que possibilita a incorporação de evidências sistematizadas por especialistas, de forma a construir um corpo de conhecimento sobre um determinado tema de relevância científica ${ }^{(4)}$.

Acrescenta-se que nos últimos anos a enfermagem tem se beneficiado com este tipo de estudo, que permite a aplicabilidade e utilização de resultados de pesquisas no cotidiano da atenção em saúde, à luz da "Prática Baseada em Evidências"(4).

O processo de revisão foi sistematizado em seis fases distintas, sendo a primeira delas relativa à elaboração da pergunta norteadora: "Qual a magnitude da VPI física e/ou sexual na gestação e quais as suas repercussões para a saúde materna?".

A segunda fase corresponde à amostragem na literatura, que procurou incluir a maior variedade possível de produtos identificados, de forma a garantir a variedade e amplitude dos resultados ${ }^{(4)}$.

Neste sentido, foi realizada seleção eletrônica na Biblioteca Regional de Medicina (BIREME), de onde foram acessadas as bases de dados das ciências da saúde em geral, LILACS (Literatura Latino-Americana e do Caribe), MEDLINE (National Library of Medicine, Estados Unidos) e PUBMED.

A busca eletrônica foi realizada através das seguintes combinações de Descritores em Ciências da Saúde (DeCS): "battered women" "pregnancy"; "violence against women" "pregnancy"; "domestic violence" "pregnancy"; "violence" "pregnancy", e se baseou na adoção dos seguintes critérios de inclusão: a indexação de estudos nas respectivas bases de dados, no período compreendido entre janeiro de 2007 e abril de 2012; nos idiomas português, inglês e espanhol. Foram definidos como critérios de exclusão: produções sem disponibilidade do texto na íntegra; tema central do estudo não relacionado à temática da VPI na gestação, focado na saúde do concepto ou na amamentação; abordagem sobre saúde mental, violência praticada pelo casal ou outros agressores; estudos conduzidos com populações específicas, tais como, mulheres refugiadas, soropositivas para HIV, entre outras; publicações referentes a manuais técnicos, cartilhas, editoriais, e 
estudos classificados em nível de evidência quatro, cinco ou seis, conforme segue especificado na análise dos estudos.

A terceira fase do estudo consistiu na coleta de dados por meio de instrumento descritivo acerca dos seguintes elementos: autoria, ano de publicação, título, periódico, país onde foi realizado o estudo, métodos e resultados.

A análise crítica dos estudos selecionados correspondeu à quarta fase do estudo. A tomada de decisão quanto à aceitação ou rejeição dos estudos foi auxiliada pela classificação dos mesmos em níveis hierárquicos de evidências, segundo metodologia utilizada na sua elaboração(4): nível 1: evidências derivadas da meta-análise de múltiplos estudos clínicos controlados e randomizados; nível 2: evidências de estudos individuais com delineamento experimental; nível 3: evidências de estudos quase experimentais; nível 4: evidências de estudos não experimentais, descritivos ou com abordagem qualitativa; nível 5: evidências provenientes de relatos de caso ou de experiência ou de avaliação de programas com qualidade verificável; nível 6: evidências baseadas em pareceres de autoridades de notório saber, comitês.

Uma primeira análise foi realizada pela leitura de títulos dos estudos selecionados, seguida pela leitura e análise crítica dos resumos, tendo sido rejeitados: a) estudos com tema central não relacionado à VPI física e/ou sexual na gestação; b) estudos realizados com populações específicas; c) estudos que permitiram a classificação como nível 4, 5 ou 6.

Em uma segunda análise, procedeu-se a verificação de conteúdos na íntegra, que foi norteada pela técnica de análise temática para identificação das ideias centrais apresentadas, como também pela classificação em níveis de evidência. Neste processo, optou-se pela exclusão de estudos focados na violência psicológica exclusiva, violência praticada por ambos os cônjuges ou outros agressores; tendo sido incluídos os estudos classificados como nível 1, 2 ou 3.

Por fim, dada a relevância e heterogeneidade dos produtos trazidos pelas produções científicas, foram realizadas novas análises críticas dos resultados obtidos. Desta forma, optou-se pelo agrupamento da amostra final de 45 produções científicas relacionadas ao tema da violência por parceiros íntimos na gestação, em duas categorias de análise dos resultados: 1) prevalência da
VPI na gestação e fatores associados; 2) repercussões da VPI sobre a saúde materna.

A Figura 1 apresenta os principais elementos do delineamento desta revisão integrativa, no que se refere às fases de amostragem, coleta de dados e análise crítica dos estudos. As próximas fases, correspondentes à discussão dos resultados ( $5^{\text {a }}$ fase) e apresentação da revisão integrativa (6 $6^{\text {fase}) ~ s e r a ̃ o ~ a b o r d a d a s ~ a ~ s e g u i r . ~}$ 
Critérios de Inclusão: estudos publicados em periódicos nacionais ou internacionais nos idiomas inglês, português e espanhol, entre janeiro de 2007 e abril de 2012, que apresentaram nível de evidência científica 1,2 ou 3.<smiles>C1CCCCCC1</smiles>

Critérios de Exclusão: estudos com tema central não relacionado à VPI física e/ou sexual na gestação; estudos realizados com populações específicas; estudos classificados como nível 4, 5 ou 6 de evidência científica.
Busca eletrônica BVS

(MEDLINE, PUBMED, LILACS)

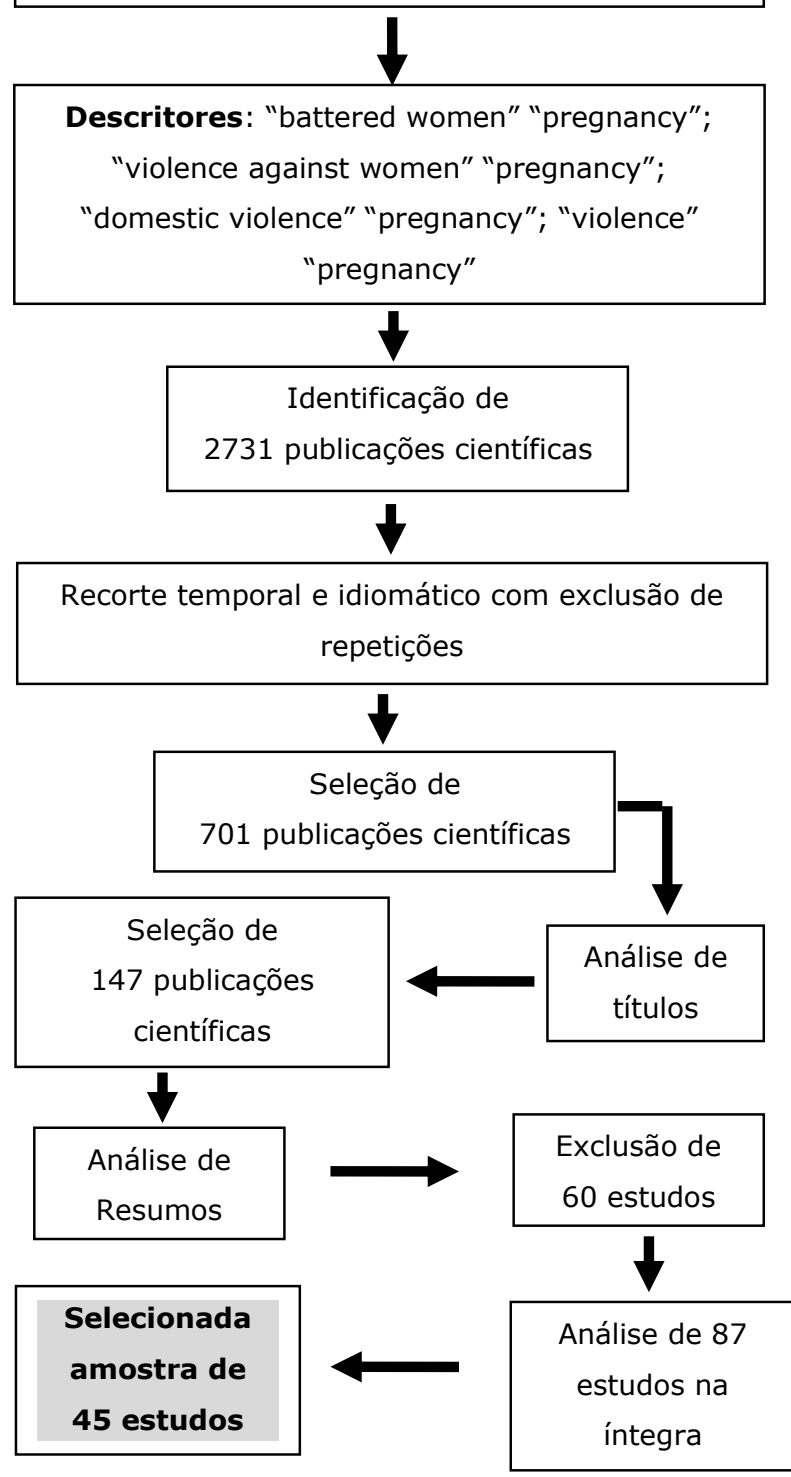

Figura 1: Representação esquemática do delineamento do estudo.

\section{RESULTADOS E DISCUSSÃO}

Houve consenso entre os 45 estudos que integram a amostra (Quadro 1) que a violência por parceiros íntimos representa um grave problema de saúde pública, dada a sua magnitude e consequências associadas à saúde reprodutiva, física e mental das mulheres. 
Quadro 1: Taxas de prevalência e consequências da VPI identificadas pelos estudos que compuseram a amostra, segundo tipo de violência, características do estudo, autores, periódico, título, ano de publicação e país onde foi realizada a investigação.

\begin{tabular}{|c|c|c|c|c|c|}
\hline \multicolumn{2}{|r|}{$\begin{array}{c}\text { AUTOR/ANO/ } \\
\text { PERIÓDICO/PAÍs }\end{array}$} & TíTULO & \multirow{2}{*}{$\begin{array}{c}\text { CARACTERÍSTICA DO ESTUDO } \\
\text { Estudo com } 104 \text { gestantes dos Apalaches (EUA) } \\
\text { entrevistadas durante o pré-natal por } \\
\text { instrumento baseado no CTS2, para verificar } \\
\text { prevalência e fatores associados à VPI. }\end{array}$} & \multirow{2}{*}{$\begin{array}{c}\text { PREVALÊNCIAS } \\
\text { VP e/ou VF e/ou VS: } \\
\text { 80,8\%; VP: } 79,8 \% ; \text { VF: } \\
\text { 27,9\%; VS: } 20,2 \% . \\
\text { Gestação atual } \\
\end{array}$} & \multirow{2}{*}{$\begin{array}{l}\text { CONSEQUÊNCIAS } \\
\text { VPI associada com tabagismo, uso } \\
\text { abusivo de álcool e drogas ilicitas, } \\
\text { início tardio do pré-natal }(p<0,05) \text {. }\end{array}$} \\
\hline 1. & $\begin{array}{l}\text { Bailey \& Daugherty, } 2007^{(12)} \\
\text { Matern Child Health J } \\
\text { EUA }\end{array}$ & $\begin{array}{l}\text { Intimate partner violence during } \\
\text { pregnancy: incidence and associated } \\
\text { health behaviors in a rural population. }\end{array}$ & & & \\
\hline 2. & $\begin{array}{l}\text { Coonrod et al., } 2007^{(8)} \\
\text { Am J Obstet Gynecol } \\
\text { EUA }\end{array}$ & $\begin{array}{c}\text { Asymptomatic bacteriuria and } \\
\text { intimate partner violence in pregnant } \\
\text { women. }\end{array}$ & $\begin{array}{c}\text { Entrevistas com } 342 \text { gestantes hispânicas de } \\
\text { baixa renda do Arizona (EUA), por meio de } \\
\text { inquérito pelo computador baseado nas escalas } \\
\text { CTS e de Castro, para verificar associação entre } \\
\text { VPI e ITU. }\end{array}$ & $\begin{array}{l}\text { VP: } 46,9 \% \\
\text { VF: } 7,6 \% \\
\text { VS: } 5,8 \% \\
\text { Gestação atual }\end{array}$ & $\begin{array}{l}\text { Não houve associação } \\
\text { estatisticamente significante entre } \\
\text { Infecção do Trato Urinário (ITU) e } \\
\text { Pielonefrite e VPI. }\end{array}$ \\
\hline 3. & $\begin{array}{c}\text { Deveci et al., } 2007 \\
\text { Southeast Asian J Trop Med } \\
\text { Public Health } \\
\text { Turquia }\end{array}$ & $\begin{array}{l}\text { Prevalence of domestic violence } \\
\text { during pregnancy in a Turkish } \\
\text { community. }\end{array}$ & $\begin{array}{l}\text { Amostra de } 249 \text { gestantes de } 02 \text { serviços de } \\
\text { saúde de Elazig, para estima prevalência de VPI } \\
\text { na gestação e fatores de risco associados. }\end{array}$ & $\begin{array}{l}\text { VP: } 30,5 \% \\
\text { VF: } 4,8 \% \\
\text { VS: } 4,4 \% . \\
\text { Gestação atual }\end{array}$ & \\
\hline 4. & $\begin{array}{l}\text { Doubova, et al., } 2007 \\
\text { Rev Saude Publica } \\
\text { México }\end{array}$ & $\begin{array}{l}\text { Violencia de pareja en mujeres } \\
\text { embarazadas en la Ciudad de México. }\end{array}$ & $\begin{array}{c}\text { Estudo transversal com } 383 \text { gestantes } \\
\text { entrevistadas no pré-natal na Cidade do México, } \\
\text { por meio de instrumento desenvolvido pelos } \\
\text { autores. }\end{array}$ & $\begin{array}{c}\text { VP e/ou VF e/ou VS: } \\
31,1 \% \\
\text { VP: } 93 \% . \\
\text { Gestação atual }\end{array}$ & \\
\hline 5. & $\begin{array}{l}\text { Durang \& Schraiber, } 2007^{(14)} \\
\text { Rev Bras Epidemiol. } \\
\text { Brasil }\end{array}$ & $\begin{array}{l}\text { Violência na gestação entre usuárias } \\
\text { de serviços públicos de saúde da } \\
\text { Grande São Paulo: prevalência e } \\
\text { fatores associados. }\end{array}$ & $\begin{array}{c}\text { Entrevistas com } 1.922 \text { usuárias de serviços de } \\
\text { saúde, entre } 15 \text { e } 49 \text { anos, para estimar a } \\
\text { prevalência da VPI na gestação e analisar os } \\
\text { fatores associados. }\end{array}$ & $\begin{array}{l}\text { VP e/ou VF e/ou VS: } \\
20 \% . \\
\text { Em alguma gestação }\end{array}$ & \\
\hline 6. & $\begin{array}{l}\text { Bourassa \& Berubé, } 2007 \\
\text { J Obstet Gynaecol Can } \\
\text { Canadá }\end{array}$ & $\begin{array}{l}\text { The Prevalence of Intimate Partner } \\
\text { Violence Among Women and } \\
\text { Teenagers Seeking Abortion } \\
\text { Compared With Those Continuing } \\
\text { Pregnancy }\end{array}$ & $\begin{array}{l}\text { Investigação sobre VPI entre } 350 \text { mulheres e } \\
\text { adolescentes que requisitaram abortamento } \\
\text { eletivo (EA), comparadas ao grupo de } 653 \\
\text { mulheres que continuaram com a gravidez (CP). }\end{array}$ & & $\begin{array}{l}\text { A probabilidade de VPI no último } \\
\text { ano foi quatro vezes maior entre as } \\
\text { mulheres do grupo abortamento } \\
\text { eletivo }(p<0,0001) \text {. }\end{array}$ \\
\hline 7. & $\begin{array}{l}\text { El-Khorazaty et al. } 2007 \\
\text { BMC Public Health } \\
\text { EUA }\end{array}$ & $\begin{array}{l}\text { Recruitment and retention of low- } \\
\text { income minority women in a } \\
\text { behavioral intervention to reduce } \\
\text { smoking, depression, and intimate } \\
\text { partner violence during pregnancy }\end{array}$ & $\begin{array}{c}\text { Gestantes de baixa renda foram avaliadas quanto } \\
\text { ao risco para depressão, tabagismo e VPI, } \\
\text { utilizando-se o Audio Computer-Assisted Self- } \\
\text { Interview. Mulheres elegíveis, selecionadas } \\
\text { aleatoriamente, completaram inquérito via } \\
\text { telefone e participaram de intervenção } \\
\text { comportamental para redução destes agravos } \\
\text { durante a gravidez. }\end{array}$ & $\begin{array}{l}\text { VP e/ou VF e/ou VS: } \\
20,9 \% \text {. } \\
\text { Gestação atual }\end{array}$ & \\
\hline 8. & $\begin{array}{l}\text { Audi et al., } 2008^{(13)} \\
\text { Rev Saude Publica } \\
\text { Brasil }\end{array}$ & $\begin{array}{l}\text { Violence against pregnant women: } \\
\text { prevalence and associated factors }\end{array}$ & $\begin{array}{l}1.379 \text { gestantes entrevistadas durante o pré- } \\
\text { natal em Campinas, através de instrumento } \\
\text { validado por Schraiber et al. (2006). }\end{array}$ & $\begin{array}{l}\text { VP: } 19,1 \% ; \text { VF e VS: } \\
\text { 6,5\%. } \\
\text { Gestação atual }\end{array}$ & \\
\hline
\end{tabular}




\begin{tabular}{|c|c|c|c|c|c|}
\hline \multicolumn{2}{|r|}{$\begin{array}{c}\text { AUTOR/ANO/ } \\
\text { PERIÓDICO/PAÍS }\end{array}$} & TíTULO & \multirow[b]{2}{*}{$\begin{array}{c}\text { CARACTERÍSTICA DO ESTUDO } \\
\text { Estudo de coorte com } 1.507 \text { mulheres nulíparas } \\
\text { após a } 24^{\text {a }} \text { semana de gravidez, que responderam } \\
\text { a um questionário e entrevistas por telefone, } \\
\text { entre a } 30^{\mathrm{a}} \text { e } 32^{\mathrm{a}} \text { semanas e } 3,6,9,12 \text { e } 18 \text { meses } \\
\text { após o parto. As duas questões sobre medo do } \\
\text { parceiro foram elaboradas pelo próprio grupo de } \\
\text { pesquisa. Foram divididos dois grupos para } \\
\text { análise: mulheres que relataram e que negaram } \\
\text { medo do parceiro. }\end{array}$} & \multirow[b]{2}{*}{$\begin{array}{c}\text { PREVALÊNCIAS } \\
\\
\text { 18,7\% tiveram medo } \\
\text { do parceiro alguma vez } \\
\text { na vida; } 3,1 \% \text { tiveram } \\
\text { medo no início da } \\
\text { gravidez e } 15,6 \% \text { após } \\
\text { a gestação. }\end{array}$} & \multirow[b]{2}{*}{$\begin{array}{c}\text { CONSEQUÊNCIAS } \\
\text { A comparação entre os grupos } \\
\text { identificou risco para incontinência } \\
\text { urinária (ORaj=1.64; IC95\%: 0.9- } \\
\text { 3.1); incontinência fecal } \\
\text { (ORaj=3.32;IC 95\%: } 1.2-9.2 \text { ); } \\
\text { sangramento vaginal (ORaj=2.84; } \\
\text { IC95\%: } 1.5-5.5) \text {; ansiedade } \\
\text { (ORaj=10.22; IC95\%: 5.0-21.2), e } \\
\text { depressão (ORaj= } \\
\text { 4.43; IC95\%:2.1-9.7) }\end{array}$} \\
\hline 9. & $\begin{array}{l}\text { Brown et al., } 2008 \\
\text { Birth } \\
\text { Australia }\end{array}$ & $\begin{array}{c}\text { Fear of an Intimate Partner and } \\
\text { Women's Health in Early Pregnancy: } \\
\text { Findings from the Maternal Health } \\
\text { Study }\end{array}$ & & & \\
\hline 10. & $\begin{array}{l}\text { Calderón et al., } 2008 \\
\text { Am J Prev Med } \\
\text { EUA }\end{array}$ & $\begin{array}{l}\text { Cueing prenatal provider's effects on } \\
\text { discussions of intimate partner. }\end{array}$ & $\begin{array}{l}\text { Mulheres a partir da } 26^{a} \text { semana de gestação. } \\
\text { Participantes que relataram um ou mais riscos } \\
\text { para tabagismo, uso abusivo de álcool ou drogas } \\
\text { e VPI foram randomizadas em dois grupos } \\
\text { (intervenção e controle). A avaliação de risco } \\
\text { para VPI baseou-se no AAS. }\end{array}$ & $\begin{array}{l}\text { VP e/ou VF e/ou VS: } \\
13 \% \text {. } \\
\text { Gestação atual. }\end{array}$ & \\
\hline 11. & $\begin{array}{l}\text { Cripe et al., } 2008 \\
\text { Int J Gynecol Obstet } \\
\text { Peru }\end{array}$ & $\begin{array}{l}\text { Association of intimate partner } \\
\text { physical and sexual violence with } \\
\text { unintended pregnancy among } \\
\text { pregnant women in Peru. }\end{array}$ & $\begin{array}{c}\text { Estudo transversal com } 2167 \text { puérperas do } \\
\text { Instituto Nacional Materno Perinatal de Lima } \\
\text { (Peru) entrevistadas durante o pós-parto, para } \\
\text { examinar a associação entre VPI e gravidez } \\
\text { indesejada. }\end{array}$ & & $\begin{array}{l}\text { O risco de gravidez indesejada foi } \\
\text { 3,31 vezes maior entre as mulheres } \\
\text { que sofreram VPI física e sexual } \\
\qquad(p<0.0005)\end{array}$ \\
\hline 12. & $\begin{array}{c}\text { Fanslow et al., } 2008 \\
\text { Aust N Z J Obstet Gynaecol. } \\
\text { Nova Zelândia }\end{array}$ & $\begin{array}{l}\text { Pregnancy outcomes and intimate } \\
\text { partner violence in New Zealand. }\end{array}$ & $\begin{array}{c}\text { Estudo quantitativo com amostra populacional } \\
\text { de } 2391 \text { mulheres entre } 18 \text { e } 64 \text { anos, que já } \\
\text { engravidaram. Investigação de VPI através de } \\
\text { questionário adaptado do Estudo Multipaíses } \\
\text { OMS. }\end{array}$ & & $\begin{array}{c}\text { VPI associada à probabilidade de } \\
\text { aborto espontâneo } 1,4 \text { vezes maior } \\
\text { ( } p=0,008) \text { e } 2,5 \text { vezes maior de } \\
\text { aborto induzido ( } p<0,0001 \text { ) entre } \\
\text { mulheres com VPI. }\end{array}$ \\
\hline 13. & $\begin{array}{l}\text { Gao et al., } 2008 \\
\text { Int J Gynaecol Obstet. } \\
\text { Nova Zelândia }\end{array}$ & $\begin{array}{l}\text { Intimate partner violence and } \\
\text { unplanned pregnancy in the Pacific } \\
\text { Islands Families Study. }\end{array}$ & $\begin{array}{c}\text { Entrevistas com } 1088 \text { puérperas selecionadas do } \\
\text { "Mothers of the Pacific Islands Families (PIF)", } \\
\text { para determinar a associação entre VPI e } \\
\text { gravidez indesejada, através de instrumento } \\
\text { baseado na escala CTS. }\end{array}$ & & $\begin{array}{c}\text { Mulheres com VPI física } \\
\text { apresentaram maior probabilidade } \\
\text { de relatar que a gravidez não foi } \\
\text { planejada }(\mathrm{OR}=1,78 ; \text { IC95\%:1,32- } \\
2,40) \text {. }\end{array}$ \\
\hline 14. & $\begin{array}{l}\text { Flores et al., } 2008^{(15)} \\
\text { Rev enferm herediana } \\
\text { México }\end{array}$ & $\begin{array}{l}\text { Violencia de género, embarazo y } \\
\text { autoestima en un área urbana de la } \\
\text { ciudad de México. }\end{array}$ & $\begin{array}{c}\text { Estudo transversal com } 490 \text { mulheres mexicanas } \\
\text { de } 15 \text { a } 45 \text { anos, entrevistadas através de } \\
\text { instrumento baseado na "Encuesta Nacional de } \\
\text { Violencia contra las Mujeres". }\end{array}$ & $\begin{array}{l}\text { VP e/ou VF e/ou VS: } \\
94 \% \\
\text { Alguma gestação }\end{array}$ & $\begin{array}{c}\text { Maiores chances de violência } \\
\text { psicológica } \\
\text { (RC=2,29; IC } 95 \%: 1,39-3,75) \text { e baixa } \\
\text { autoestima }(R C=1,06 ; \text { IC } 95 \%: 1,63- \\
2,78) .\end{array}$ \\
\hline 15. & $\begin{array}{l}\text { Morland et al., } 2008 \\
\text { J Interpers Violence. } \\
\text { EUA }\end{array}$ & $\begin{array}{l}\text { Intimate partner violence and } \\
\text { miscarriage: examination of the role } \\
\text { of physical and psychological abuse } \\
\text { and postraumatic stress disorder. }\end{array}$ & $\begin{array}{l}\text { Análise secundária do banco de Estudo de Risco } \\
\text { da Saúde da Mulher (CWHRS), sobre a relação } \\
\text { entre VPI e aborto espontâneo de } 119 \text { mulheres } \\
\text { entre } 705 \text { que participaram do CWHRS. VPI foi } \\
\text { avaliada pela Power and Control Scale. }\end{array}$ & & $\begin{array}{l}\text { Aborto: Média de escores entre as } \\
\text { mulheres que sofreram VF severa e } \\
\text { que também desenvolveram TEPT } \\
\text { foi maior entre as mulheres que } \\
\text { abortaram espontaneamente. }\end{array}$ \\
\hline
\end{tabular}




\begin{tabular}{|c|c|c|c|c|c|}
\hline & $\begin{array}{l}\text { AUTOR/ANO/ } \\
\text { PERIÓDICO/PAÍS }\end{array}$ & TíTULO & CARACTERÍSTICA DO ESTUDO & PREVALÊNCIAS & CONSEQUÊNCIAS \\
\hline 16. & $\begin{array}{c}\text { Roelens et al., } 2008 \\
\text { Eur J Obstet Gynecol Reprod } \\
\text { Biol } \\
\text { Bélgica }\end{array}$ & $\begin{array}{c}\text { Disclosure and health-seeking } \\
\text { behavior following intimate partner } \\
\text { violence before and during pregnancy } \\
\text { in Flanders, Belgium: a survey } \\
\text { surveillance study. }\end{array}$ & $\begin{array}{c}\text { Estudo multicêntrico com } 537 \text { gestantes de cinco } \\
\text { hospitais da Bélgica. Dados coletados de } \\
\text { questionário anônimo respondido pelas } \\
\text { gestantes e enviados a equipe de pesquisa, } \\
\text { baseado no AAS. }\end{array}$ & $\begin{array}{l}\text { VP e/ou VF e/ou VS: } \\
3,4 \% \text {. } \\
\text { Gestação atual e/ou } \\
\text { último ano }\end{array}$ & \\
\hline 17. & $\begin{array}{c}\text { Sanchez et al., } 2008^{(5)} \\
\text { Eur J Obstet Gynecol Reprod } \\
\text { Biol } \\
\text { Peru }\end{array}$ & $\begin{array}{l}\text { Intimate partner violence (IPV) and } \\
\text { preeclampsia among Peruvian women. }\end{array}$ & $\begin{array}{c}\text { Estudo caso-controle em um hospital de Lima } \\
\text { (Peru) para examinar a relação entre VPI na } \\
\text { gestação e risco para pré-eclampsia. Instrumento } \\
\text { desenvolvido pelos autores. }\end{array}$ & $\begin{array}{c}\text { VP e/ou VF e/ou } \\
\text { Vs: } 24,3 \% \text { (controles) e } \\
43,1 \% \text { (casos). } \\
\text { Gestação atual }\end{array}$ & $\begin{array}{l}\text { Mulheres que relataram VPI } \\
\text { apresentaram risco } 2,4 \text { vezes maior } \\
\text { de pré-eclampsia }(\mathrm{OR}=2,4 ; \\
\text { IC95\%:1,7-3,3). }\end{array}$ \\
\hline 18. & $\begin{array}{c}\text { Thananowan, } 2008 \\
\text { Violence Against Women } \\
\text { Tailandia }\end{array}$ & $\begin{array}{l}\text { Intimate Partner Violence Among } \\
\text { Pregnant Thai Women }\end{array}$ & $\begin{array}{c}\text { Estudo transversal com } 475 \text { gestantes de } 5 \\
\text { serviços de pré-natal de Bangkok, entrevistadas } \\
\text { por instrumento baseado no AAS. }\end{array}$ & $\begin{array}{l}\text { VF: } 4,8 \% . \\
\text { Gestação atual }\end{array}$ & \\
\hline 19. & $\begin{array}{l}\text { Perales et al., } 2009^{(10)} \\
\text { Violence Against Women. } \\
\text { Peru }\end{array}$ & $\begin{array}{l}\text { Prevalence, types, and pattern of } \\
\text { intimate partner violence among } \\
\text { pregnant women in Lima, Peru. }\end{array}$ & $\begin{array}{c}\text { Estudo transversal com } 2.392 \text { puérperas. } \\
\text { Abordagem sobre VPI foi adaptada do Módulo de } \\
\text { Violência Doméstica do Inquérito Demográfico } \\
\text { de Saúde de } 2005 \text { e do Estudo Multipaíses } \\
\text { OMS }^{28} \text {. }\end{array}$ & $\begin{array}{l}\text { VP, VF e VS: } 9,2 \% \\
\text { VP: } 37,8 \% ; \\
\text { VF: } 21,0 \% \\
\text { VS: } 4,1 \% . \\
\text { Gestação atual }\end{array}$ & \\
\hline 20. & $\begin{array}{l}\text { Valladares et al., } 2009 \text { (20) } \\
\text { Acta Obstet Gynecol Scand. } \\
\text { Suíça/Nicarágua }\end{array}$ & $\begin{array}{l}\text { Neuroendocrine response to violence } \\
\text { during pregnancy--impact on duration } \\
\text { of pregnancy and fetal growth. }\end{array}$ & $\begin{array}{c}\text { Estudo de base comunitária, com } 147 \text { gestantes } \\
\text { de Leon (Nicarágua), para as quais foram } \\
\text { aplicadas escalas padronizadas para medir VPI, } \\
\text { estresse e condições socioeconômicas. }\end{array}$ & $\begin{array}{l}\text { VP: } 44 \% \\
\text { VF: } 17 \% \\
\text { VS: } 8 \% . \\
\text { Gestação atual }\end{array}$ & $\begin{array}{l}\text { Estresse materno na gestação, } \\
\text { associado à redução de peso } \\
\text { infantil ao nascer }(p=0,001) \text {. }\end{array}$ \\
\hline 21. & $\begin{array}{c}\text { Kothari et al., } 2009 \\
\text { J Womens Health (Larchmt.) } \\
\text { EUA }\end{array}$ & $\begin{array}{l}\text { Perinatal Status and Help-Seeking for } \\
\text { Intimate Partner Violence }\end{array}$ & $\begin{array}{c}\text { Análise retrospectiva dos arquivos médicos e } \\
\text { policiais de } 964 \text { mulheres que sofreram VPI em } \\
\text { 2000. Foram comparados dois grupos distintos: } \\
\text { perinatal e não-perinatal. }\end{array}$ & & $\begin{array}{c}\text { Mulheres abusadas apresentaram } \\
\text { duas vezes mais chances de } \\
\text { gravidez }(p<0.001) . \text { Status perinatal } \\
\text { aumentou procura por apoio } \\
\text { judicial }(p<0.01) \text {. }\end{array}$ \\
\hline 22. & $\begin{array}{l}\text { Chan et al., } 2009 \\
\text { J Interp Violence } \\
\text { China }\end{array}$ & $\begin{array}{c}\text { Correlates of In-Law Conflict and } \\
\text { Intimate Partner Violence Against } \\
\text { Chinese Pregnant Women in Hong } \\
\text { Kong }\end{array}$ & $\begin{array}{l}3245 \text { gestantes recrutadas em sete hospitais } \\
\text { foram investigadas sobre VPI a partir do AAS. }\end{array}$ & $\begin{array}{l}\text { VP e/ou VF e/ou VS: } \\
9,1 \% . \\
\text { VP: } 6,7 \% \\
\text { VF ou VS: } 2,5 \% \text {. Último } \\
\text { ano. } \\
\end{array}$ & \\
\hline 23. & $\begin{array}{l}\text { Eisenman et al., } 2009 \\
\text { Violence Vict. } \\
\text { EUA }\end{array}$ & $\begin{array}{c}\text { Intimate Partner Violence and } \\
\text { Community Service Needs among } \\
\text { Pregnant and Post-partum Latina } \\
\text { Women }\end{array}$ & $\begin{array}{l}\text { Foi examinada a relação entre VPI na gestação e } \\
\text { procura por serviços sociais, legais e empregos, } \\
\text { através de uma coorte de } 210 \text { gestantes latinas. }\end{array}$ & & $\begin{array}{l}\text { Gestantes que relataram VPI } \\
\text { buscaram mais serviços legais } \\
(p=0.006) \text { e sociais }(p<0,05) \text {. }\end{array}$ \\
\hline 24. & $\begin{array}{l}\text { Kim et al., } 2010^{(6)} \\
\text { Med Health RI } \\
\text { EUA }\end{array}$ & $\begin{array}{l}\text { Intimate Partner Violence Before or } \\
\text { During Pregnancy In Rhode Island }\end{array}$ & $\begin{array}{l}\text { Estudo de base populacional, que analisou VPI } \\
\text { antes e durante a gravidez, com } 5.662 \text { puérperas. }\end{array}$ & $\begin{array}{l}\text { VP ou VF ou VS: } \\
3,2 \% \text {. Gestação atual }\end{array}$ & \\
\hline
\end{tabular}




\begin{tabular}{|c|c|c|c|c|c|}
\hline \multicolumn{2}{|r|}{$\begin{array}{c}\text { AUTOR/ANO/ } \\
\text { PERIÓDICO/PAÍS }\end{array}$} & \multirow{2}{*}{$\begin{array}{c}\text { TíTULO } \\
\text { MOSAIC (Mothers' Advocates In the } \\
\text { Community): protocol and sample } \\
\text { description of a cluster randomized } \\
\text { trial of mentor mother support to } \\
\text { reduce intimate partner violence } \\
\text { among pregnant or recent mothers }\end{array}$} & \multirow{2}{*}{$\begin{array}{c}\text { CARACTERÍSTICA DO ESTUDO } \\
\text { Gestantes ou mulheres com filhos pequenos, } \\
\text { moradoras do subúrbio, identificadas como } \\
\text { abusadas ou sintomáticas para VPI, através do } \\
\text { Composite Abuse Scale (CAS), foram } \\
\text { encaminhadas para intervenção de enfermagem. }\end{array}$} & \multirow[t]{2}{*}{ PREVALÊNCIAS } & \multirow[t]{2}{*}{ CONSEQUÊNCIAS } \\
\hline 25. & $\begin{array}{l}\text { Taft et al., } 2009 \\
\text { BMC Public Health } \\
\text { Australia }\end{array}$ & & & & \\
\hline 26. & $\begin{array}{l}\text { Salazar et al., } 2009^{(23)} \\
\text { BMC Public Health } \\
\text { Nicaragua }\end{array}$ & $\begin{array}{l}\text { Ending Intimate Partner Violence } \\
\text { after pregnancy: Findings from a } \\
\text { community-based longitudinal study } \\
\text { in Nicaragua }\end{array}$ & $\begin{array}{c}\text { Estudo longitudinal com } 478 \text { gestantes } \\
\text { recrutadas do León Health and Demographic } \\
\text { Surveillance System baseline 2002-2003 (HDSS), } \\
\text { investigadas sobre VPI por instrumento do } \\
\text { Estudo Multipaíses OMS durante e após a } \\
\text { gravidez. }\end{array}$ & $\begin{array}{l}\text { VP e/ou VF e/ou VS: } \\
\text { 32,1\%. Gestação atual. }\end{array}$ & \\
\hline 27. & $\begin{array}{l}\text { Cripe et al., } 2010 \\
\text { J Interpers Violence } \\
\text { Peru }\end{array}$ & $\begin{array}{l}\text { Intimate Partner Violence During } \\
\text { Pregnancy: A Pilot Intervention } \\
\text { Program in Lima, Peru }\end{array}$ & $\begin{array}{l}\text { Gestantes entre a 12a e 26a semanas foram } \\
\text { avaliadas quanto ao risco para VPI pelo AAS, } \\
\text { randomizadas em dois grupos de estudo: } \\
\text { intervenção padrão e intervenção } \\
\text { empoderamento. Após, foram reavaliadas pelo } \\
\text { CTS2 ( } n=204) \text {. }\end{array}$ & $\begin{array}{c}\text { VP: } 42,2 \% . \text { Gestação } \\
\text { atual. }\end{array}$ & \\
\hline 28. & $\begin{array}{l}\text { Chu et al., } 2010^{(9)} \\
\text { Am J Prev Med } \\
\text { EUA }\end{array}$ & $\begin{array}{l}\text { Physical Violence Against U.S. Women } \\
\text { Around the Time of Pregnancy, 2004- } \\
2007 .\end{array}$ & $\begin{array}{c}\text { Entrevistas com } 134.955 \text { puérperas americanas a } \\
\text { partir de um questionário enviado pelo correio, } \\
\text { contendo quatro questões sobre violência física } \\
\text { durante a gravidez. }\end{array}$ & $\begin{array}{c}\text { VF: } 3,6 \% . \\
\text { Gestação atual }\end{array}$ & \\
\hline 29. & $\begin{array}{l}\text { Leone et al., } 2010^{(18)} \\
\text { J Womens Health (Larchmt.) } \\
\text { EUA }\end{array}$ & $\begin{array}{c}\text { Effects of Intimate Partner Violence } \\
\text { on Pregnancy Trauma and Placental } \\
\text { Abruption }\end{array}$ & $\begin{array}{l}\text { Estudo para examinar a associação entre VPI, } \\
\text { trauma pré-natal e descolamento prematuro de } \\
\text { placenta a partir da análise de registros } \\
\text { hospitalares e de atendimento pré-natal de } 2873 \\
\text { gestantes. O instrumento de avaliação de VPI foi } \\
\text { desenvolvido pelo próprio programa SHS. }\end{array}$ & $\begin{array}{l}\text { VP ou VF ou VS: } \\
\quad 3,7 \% \text {. } \\
\text { Gestação atual }\end{array}$ & $\begin{array}{l}\text { Gravidez Indesejada e Aborto } \\
\qquad(p<0,05) \text {. } \\
\text { Trauma na gravidez ( } p<0,01) . \\
\text { Descolamento Prematuro de } \\
\text { Placenta }(p<0,05) \text {. Gravidez } \\
\text { Indesejada }(p<0,05) \text {, entre as } \\
\text { gestantes que relataram VPI. }\end{array}$ \\
\hline 30. & $\begin{array}{l}\text { Li et al., } 2010 \\
\text { Am J Public Health } \\
\text { EUA }\end{array}$ & $\begin{array}{l}\text { A Multilevel Analysis of Individual, } \\
\text { Household and Neighborhood } \\
\text { Correlates of Intimate Partner } \\
\text { Violence Among Low-Income } \\
\text { Pregnant Women in Alabama. }\end{array}$ & $\begin{array}{l}\text { Estudo multinível para investigar VPI entre } 2887 \\
\text { gestantes do Alabama (EUA). VPI investigada por } \\
\text { instrumento baseado no AAS. }\end{array}$ & $\begin{array}{c}\text { VF ou VS: } 7,4 \% \text { Último } \\
\text { ano }\end{array}$ & $\begin{array}{l}\text { Uso de álcool apresentou-se } \\
\text { associado à VPI (OR=2.54; } \\
\text { IC95\%:1.38-4.68, } p=0,003)\end{array}$ \\
\hline 31. & $\begin{array}{l}\text { Silverman et al., } 2010 \\
\text { Am J Public Health } \\
\text { EUA }\end{array}$ & $\begin{array}{c}\text { Male Perpetration of Intimate Partner } \\
\text { Violence and Involvement in } \\
\text { Abortions and Abortion- Related } \\
\text { Conflict }\end{array}$ & $\begin{array}{c}\text { Estudo com } 1318 \text { homens entre } 18 \text { e } 35 \text { anos, } \\
\text { recrutados em três serviços de saúde de áreas } \\
\text { urbanas com população de baixa renda. Os } \\
\text { homens forma investigados sobre VPI através de } \\
\text { uma versão adaptada do CTS2. }\end{array}$ & $\begin{array}{l}\text { VF ou VS: } 39,1 \% \text {. } \\
\text { Alguma vez na vida, } \\
\text { contra parceira do } \\
\text { sexo feminino. }\end{array}$ & $\begin{array}{l}\text { 33,2\% estiveram envolvidos em } \\
\text { gestações que terminaram em } \\
\text { aborto provocado. }\end{array}$ \\
\hline
\end{tabular}




\begin{tabular}{|c|c|c|c|c|c|}
\hline \multicolumn{2}{|r|}{$\begin{array}{l}\text { AUTOR/ANO/ } \\
\text { PERIÓDICO/PAÍS }\end{array}$} & TíTULO & CARACTERÍSTICA DO ESTUDO & PREVALÊNCIAS & CONSEQUÊNCIAS \\
\hline 32. & $\begin{array}{l}\text { Oweis et al., } 2010 \\
\text { Matern Child Health J } \\
\text { Jordânia }\end{array}$ & $\begin{array}{c}\text { Prevalence of Violence During } \\
\text { Pregnancy: Findings from a Jordanian } \\
\text { Survey }\end{array}$ & $\begin{array}{l}\text { Estudo descritivo para estimar a prevalência de } \\
\text { VPI na gestação com } 316 \text { gestantes, } \\
\text { entrevistadas por meio de instrumento } \\
\text { formulado pela equipe de pesquisa. }\end{array}$ & $\begin{array}{l}\text { VP: } 23,4 \% \\
\text { VF: } 10,4 \% \\
\text { VS: } 5,7 \% . \\
\text { Gestação atual }\end{array}$ & \\
\hline 33. & $\begin{array}{l}\text { Beydoun et al., } 2010^{(21)} \\
\text { Ann Epidemiol } \\
\text { Canadá }\end{array}$ & $\begin{array}{l}\text { Intimate Partner Violence as a Risk } \\
\text { Factor for Postpartum Depression } \\
\text { Among Canadian Women in the } \\
\text { Maternity Experience Survey }\end{array}$ & $\begin{array}{l}\text { Estudo de base populacional com } 6421 \\
\text { puérperas canadenses entrevistadas por } \\
\text { telefone assistido por computador, a partir da } \\
\text { Escala de Depressão Pós-parto de Edimburgo. }\end{array}$ & & $\begin{array}{l}\text { Odds ratio ajustado de depressão } \\
\text { pós-parto foi maior entre as } \\
\text { mulheres com VPI nos últimos dois } \\
\text { anos, (ORaj=1.61; IC95\%:1.06-2.45). }\end{array}$ \\
\hline 34. & $\begin{array}{l}\text { Saftlas et al., } 2010 \\
\text { Am J Public Health } \\
\text { EUA }\end{array}$ & $\begin{array}{c}\text { Prevalence of Intimate Partner } \\
\text { Violence Among an Abortion Clinic } \\
\text { Population }\end{array}$ & $\begin{array}{l}\text { Investigação sobre VPI entre } 986 \text { mulheres que } \\
\text { tiveram aborto eletivo, através de questionário } \\
\text { eletrônico, autoadministrado, baseado no } \\
\text { Women's Experience With Battering Scale. }\end{array}$ & $\begin{array}{l}\text { VP: } 9,9 \% \\
\text { VF: } 8,4 \% \\
\text { VS: } 2,5 \% . \\
\text { Último ano. }\end{array}$ & \\
\hline 35. & $\begin{array}{l}\text { Chan et al., } 2011^{(11)} \\
\text { J Interpers Violence } \\
\text { China }\end{array}$ & $\begin{array}{l}\text { Associating pregnancy with partner } \\
\text { violence against Chinese women. }\end{array}$ & $\begin{array}{c}\text { Dados do inquérito domiciliar de } 2004, \\
\text { em Hong Kong, com } 2.225 \text { homens para estimar } \\
\text { prevalência de autorelato de violência dos } \\
\text { homens contra suas parceiras. }\end{array}$ & $\begin{array}{l}\text { VF: } 11,9 \% ; \text { VS: } 9,1 \% \\
\text { VP e/ou VF e/ou } \\
\text { VS: } 18,8 \% . \\
\text { Gestação atual }\end{array}$ & $\begin{array}{c}\text { Associação significante entre } \\
\text { controle masculino na relação e } \\
\text { VPI psicológica, física e/ou sexual na } \\
\text { gestação. }\end{array}$ \\
\hline 36. & $\begin{array}{l}\text { Beydoun et al., } 2011^{(19)} \\
\text { Soc Sci Med } \\
\text { EUA }\end{array}$ & $\begin{array}{l}\text { Association of physical violence by an } \\
\text { intimate partner around the time of } \\
\text { pregnancy with inadequate } \\
\text { gestational weight gain. } \\
\end{array}$ & $\begin{array}{c}\text { Estudo transversal com dados do Oklahoma } \\
\text { (EUA) Pregnancy Risk Assessment Monitoring } \\
\text { Survey (PRAMS), para investigar associação entre } \\
\text { VPI e ganho de peso gestacional. } \\
\end{array}$ & $\begin{array}{l}\text { VP e/ou VF e/ou } \\
\text { VS:6,5\%. } \\
\text { Gestação atual e/ou } \\
\text { último ano } \\
\end{array}$ & $\begin{array}{c}\text { Associação significante entre VPI } \\
\text { física na gestação e ganho de peso } \\
\text { insuficiente, entre gestantes acima } \\
\text { de } 35 \text { anos de idade. }\end{array}$ \\
\hline 37. & $\begin{array}{l}\text { Brownridge et al., 2011 } \\
\text { Violence Against Women } \\
\text { Canada }\end{array}$ & $\begin{array}{l}\text { Pregnancy and intimate partner } \\
\text { violence: risk factors, severity, and } \\
\text { health effects. }\end{array}$ & $\begin{array}{c}\text { Estudo com } 12.300 \text { mulheres acima de } 18 \text { anos, } \\
\text { participantes do Statistics Canada's Violence } \\
\text { Against Women Survey (1993), entrevistadas por } \\
\text { telefone, através de instrumento adaptado do } \\
\text { CTS. }\end{array}$ & $\begin{array}{l}\text { VP e/ou VF e/ou VS: } \\
11,3 \% \text {. } \\
\text { Gestação atual. }\end{array}$ & $\begin{array}{c}\text { Gestantes vitimadas apresentam } 2 \\
\text { vezes mais chances de desenvolver } \\
\text { sintomas psíquicos e/ou fazer uso } \\
\text { abusivo de álcool e drogas. }\end{array}$ \\
\hline 38. & $\begin{array}{l}\text { Gartland et al., } 2011 \\
\text { Matern Child Health Jl } \\
\text { Australia }\end{array}$ & $\begin{array}{l}\text { Intimate partner violence during } \\
\text { pregnancy and the first year } \\
\text { postpartum in an Australian } \\
\text { pregnancy cohort study. }\end{array}$ & $\begin{array}{c}\text { Estudo de coorte durante e após a gravidez para } \\
\text { investigar fatores sociais e econômicos, } \\
\text { investigou } 1507 \text { gestantes nulíparas na } 15^{\text {a }} \\
\text { semana e } 3,6 \text { e } 12 \text { meses pós-parto, através de } \\
\text { questionário auto-administrado, elaborado pelos } \\
\text { autores do estudo. }\end{array}$ & $\begin{array}{l}\text { VP e/ou VF: } 17 \% \text {. No } \\
\text { primeiro ano pós- } \\
\text { parto. }\end{array}$ & \\
\hline 39. & $\begin{array}{l}\text { Johri et al., } 2011^{(7)} \\
\text { BMC Pregnancy Childbirth } \\
\text { Guatemala }\end{array}$ & $\begin{array}{l}\text { Increased risk of miscarriage among } \\
\text { women experiencing physical or } \\
\text { sexual intimate partner violence } \\
\text { during pregnancy in Guatemala City, } \\
\text { Guatemala: cross-sectional study. }\end{array}$ & $\begin{array}{c}\text { Entrevistas com } 1897 \text { gestantes atendidas em } \\
\text { um hospital de referência da Guatemala, através } \\
\text { de questionário adaptado do Estudo Multipaíses } \\
\text { OMS. }\end{array}$ & $\begin{array}{l}\text { VP e/ou VF e/ou VS: } \\
18 \% . \\
\text { VP: } 16 \% \\
\text { VF: } 10 \% \\
\text { VS: } 3 \% \text {. } \\
\text { Gestação atual } \\
\end{array}$ & $\begin{array}{l}\text { VPI associada com aborto } \\
\text { espontâneo (ORaj=1.1 -2.8). }\end{array}$ \\
\hline 40. & $\begin{array}{l}\text { Martin \& Garcia, } 2011 \\
\text { J Interpers Violence } \\
\text { EUA }\end{array}$ & $\begin{array}{l}\text { Unintended pregnancy and intimate } \\
\text { partner violence before and during } \\
\text { pregnancy among latina women in Los } \\
\text { Angeles, California. }\end{array}$ & $\begin{array}{c}\text { Entrevistas com } 313 \text { gestantes latinas para } \\
\text { verificar associação entre gravidez indesejada e } \\
\text { VPI antes e durante a gravidez, através do } \\
\text { Acculturation Rating Scale for Mexican } \\
\text { Americans (ARSMA-II). }\end{array}$ & $\begin{array}{c}\text { VP: } 19 \% \\
\text { VF: } 10 \% \\
\text { Gestação atual }\end{array}$ & $\begin{array}{l}\text { Gravidez indesejada associada à } \\
\text { VPI na gestação (ORaj= 2.79; } \\
\text { IC95\%:0.98- 7.92). }\end{array}$ \\
\hline
\end{tabular}

Rev. Eletr. Enf. [Internet]. 2012 oct/dec;14(4):944-56. Available from: http://www.fen.ufg.br/revista/v14/n4/v14n4a24.htm. 


\begin{tabular}{|c|c|c|c|c|c|}
\hline \multicolumn{2}{|r|}{$\begin{array}{c}\text { AUTOR/ANO/ } \\
\text { PERIÓDICO/PAÍS }\end{array}$} & \multirow{2}{*}{$\begin{array}{c}\text { TíTULO } \\
\text { Physical violence by partner during } \\
\text { pregnancy and use of prenatal care in } \\
\text { rural India }\end{array}$} & \multirow{2}{*}{$\begin{array}{c}\text { CARACTERÍSTICA DO ESTUDO } \\
\text { Inquérito populacional indiano com } 2,877 \\
\text { mulheres entre } 19 \text { e } 43 \text { anos, que tiveram ao } \\
\text { menos um parto. Questões sobre VPI formuladas } \\
\text { pelos autores do estudo. }\end{array}$} & \multirow{2}{*}{$\begin{array}{l}\text { PREVALÊNCIAS } \\
\text { VP e/ou VF e/ou VS: } \\
23 \% \\
\text { Alguma gestação }\end{array}$} & \multirow{2}{*}{$\begin{array}{c}\text { CONSEQUÊNCIAS } \\
\text { Baixa adesão ao pré-natal } \\
\text { (OR=0.80; IC95\%:0.68-0.95). Início } \\
\text { do pré-natal no } 3^{\circ} \text { trimestre } \\
\text { (RR=1.62; IC } 95 \%: 1.08-2.45) .\end{array}$} \\
\hline 41. & $\begin{array}{l}\text { Roski et al., } 2011^{(17)} \\
\text { J Health Popul Nutr } \\
\text { EUA }\end{array}$ & & & & \\
\hline 42. & $\begin{array}{l}\text { Roth et al., } 2011 \\
\text { Contraception } \\
\text { EUA }\end{array}$ & $\begin{array}{c}\text { Predictors of intimate partner } \\
\text { violence in women seeking medication } \\
\text { abortion }\end{array}$ & $\begin{array}{l}\text { Estudo com } 1128 \text { mulheres participantes do } \\
\text { estudo multicêntrico sobre aborto, entrevistadas } \\
\text { por questionário AAS modificado. }\end{array}$ & $\begin{array}{l}\text { VP e/ou VF e/ou VS: } \\
23 \% \\
\text { Gestação atual }\end{array}$ & $\begin{array}{l}\text { Mulheres expostas à VPI } \\
\text { apresentam maiores chances de } \\
\text { aborto espontâneo ou induzido. }\end{array}$ \\
\hline 43. & $\begin{array}{l}\text { Silva et al., } 2011^{(22)} \\
\text { Rev Saude Publica } \\
\text { Brasil }\end{array}$ & $\begin{array}{l}\text { Frequência e padrão da violência por } \\
\text { parceiro íntimo antes, durante e } \\
\text { depois da gravidez. }\end{array}$ & $\begin{array}{c}\text { Estudo de coorte, com } 960 \text { gestantes ou } \\
\text { puérperas de } 18 \text { a } 49 \text { anos, de Recife (PE). } \\
\text { Questionário adaptado do Estudo Multipaíses } \\
\text { OMS, para avaliar padrão de VPI. }\end{array}$ & $\begin{array}{l}\text { VP e/ou VF e/ou VS: } \\
31 \% \text {. } \\
\text { VP: } 28,8 \% \\
\text { VF: } 11,6 \% \\
\text { VS: } 5,6 \% \text {. } \\
\text { Gestação atual }\end{array}$ & \\
\hline 44. & $\begin{array}{l}\text { Stockl et al., 2012(1) } \\
\text { BMC Pregnancy Childbirth } \\
\text { Tanzania }\end{array}$ & $\begin{array}{l}\text { Induced abortion, pregnancy loss and } \\
\text { intimate partner violence in Tanzania: } \\
\text { a population based study. }\end{array}$ & $\begin{array}{c}\text { Análise dos dados da Tanzânia no Estudo } \\
\text { Multipaíses sobre Saúde da Mulher e Violência } \\
\text { Doméstica da OMS. }\end{array}$ & $\begin{array}{l}\text { VF e/ou VS: } 56 \% \\
\text { Alguma gestação }\end{array}$ & $\begin{array}{c}\text { Maior chance de perda da gravidez } \\
\text { (RC=1,6; IC } 95 \%: 1.06-1.60) \text { e de } \\
\text { aborto induzido (RC=1,9; } \\
\text { IC } 95 \%: 1.30 \text { - } 2.89 \text { ) entre mulheres } \\
\text { com VPI. }\end{array}$ \\
\hline 45. & $\begin{array}{c}\text { Stockl et al., 2012(2) } \\
\text { Acta Obstet Gynecol Scand } \\
\text { Alemanha }\end{array}$ & $\begin{array}{l}\text { Intimate partner violence and its } \\
\text { association with pregnancy loss and } \\
\text { pregnancy planning. }\end{array}$ & $\begin{array}{c}\text { Estudo transversal conduzido em uma } \\
\text { maternidade de Munique, para verificar } \\
\text { associação entre VPI, gravidez não planejada e } \\
\text { perda gestacional, através de instrumento } \\
\text { baseado no AAS. }\end{array}$ & $\begin{array}{c}\text { VF: } 4 \% \\
\text { Gestação atual }\end{array}$ & $\begin{array}{c}\text { Abuso físico por parceiro íntimo } \\
\text { associado à perda gestacional } \\
\text { (OR= 8.33; IC 95\%: 2,01-34,59) e } \\
\text { gravidez não planejada (OR= 5.03; } \\
\text { IC95\%:1,21-21,26). }\end{array}$ \\
\hline
\end{tabular}


Observou-se que as taxas de prevalência de VPI (psicológica e/ou física e/ou sexual) na gestação atual são variáveis entre $3,2 \%$ até $43,1 \%{ }^{(5-6)}$, dependendo da metodologia utilizada e do país onde foi realizado o estudo, além de fatores culturais, socioeconômicos e demográficos.

Considerando-se a tipificação da violência, observaram-se taxas elevadas e variáveis de violência psicológica na gestação atual entre $16 \%$ a 46,9\%(7-8) enquanto que a violência física variou entre $3,6 \%$ e $21 \%{ }^{(9-10)}$ e a forma sexual entre $3 \%$ e $9,1 \%{ }^{(7,11)}$.

A despeito das evidências disponíveis, estudo realizado no sul dos Apalaches com 104 gestantes identificou as maiores taxas de prevalência de VPI na gestação $(80,8 \%)$, correspondentes a $79,8 \%$ de violência psicológica, $27,9 \%$ de violência física e $20,2 \%$ de violência sexual, tendo sido consideradas as ocorrências nos últimos 12 meses, incluindo-se o período da gestação(12).

No Brasil, a taxa de VPI, identificada entre gestantes atendidas em unidades básicas de saúde de Campinas (SP) foi de 19,1\% de violência psicológica, e 6,5\% de violência física/sexual(13). Por outro lado, Durang \& Schraiber ${ }^{(14)}$ identificaram que $20 \%$ das 1.922 mulheres entre 15 e 49 anos entrevistadas em 14 serviços públicos de saúde do município de São Paulo, referiram algum episódio de VPI na gestação.

Entre os principais fatores associados ao rastreamento e identificação de casos de VPI na gestação que foram descritos, destacaram-se: início da vida sexual antes dos 19 anos, ser solteira ou não coabitar com o parceiro, história pregressa de violência ou abuso sexual na infância, baixo nível de escolaridade e condições econômicas desfavoráveis(12-13), baixa autoestima $^{(15)}$, controle masculino na relação(11), hábitos prejudiciais à saúde como tabagismo, abuso de álcool e drogas $(12,16)$, início tardio do acompanhamento prénatal(12,17), falta de aderência às consultas de prénatal ${ }^{(12,17)}$ e sintomas psíquicos ${ }^{(12,16)}$.

Ocorrência de VPI na gestação também se mostrou estatisticamente significante para os desfechos de aborto espontâneo ou induzido e gravidez indesejada(18), perda gestacional, ganho de peso insuficiente ${ }^{(19)}$, descolamento prematuro de placenta e trauma na gravidez ${ }^{(18)}$, pré-eclampsia(5), estresse materno associado à redução de peso infantil ao nascer ${ }^{(20)}$, sintomas psíquicos $^{(16)}$, depressão pós-parto(21), e maiores chances de violência psicológica(15).

Desta forma, alguns estudos(11,22) sugerem que a gravidez represente um fator de risco para a violência doméstica, que pode ter início na gestação ou alterar o padrão quanto à frequência e gravidade neste período, o que leva alguns autores a incluir a gravidez entre as principais causas de violência doméstica. Além disto, a VPI pode continuar a ocorrer no pós-parto, pois a gestante invariavelmente permanece ao lado do seu agressor ${ }^{(22)}$.

Também se apreende desta análise, que as características e condições de vida desfavoráveis das gestantes submetidas à VPI, podem ser mais facilmente compreendidas a partir da análise de gênero(14-15,22-23).

Privadas de autonomia para o exercício pleno de funções sociais como acesso à educação, ao trabalho remunerado, aos serviços de saúde e demais políticas públicas, as mulheres em geral, submetem-se a uma hierarquia de poder masculino, histórica e culturalmente constituída, em especial, nos países com piores indicadores de desenvolvimento humano e social(1).

Desta forma, a violência contra a mulher, se reproduz a partir das relações assimétricas de poder entre os sexos, fundamentadas em papéis sociais assumidos por homens e mulheres nas sociedades ao longo da história, e que determinam a subordinação e a opressão feminina, com base em sua função reprodutiva ${ }^{(24)}$.

Portanto, a violência na gestação torna-se tão comum quanto as demais condições rastreadas normalmente no pré-natal ${ }^{(1,23)}$. Estudos de períodos anteriores ao da pesquisa dão conta de uma prevalência de violência física em gestantes, entre 03 a $11 \%$ em países desenvolvidos e entre 04 a 32\% em países em desenvolvimento(1). Estas diferentes taxas de prevalência sugerem que as normas culturais de cada país, em certa medida, podem influenciar no grau relativo de proteção que a fase da gravidez possa assegurar contra os atos violentos.

Por outro lado, entende-se que o acesso a recursos sociais e mudanças de atitude das mulheres para não tolerar abusos e o poder de controle do parceiro, podem interferir na redução das taxas de violência na gestação(15,23).

Acredita-se que as consequências da VPI sobre a saúde materna identificadas neste estudo podem ser 
utilizadas como marcadores de risco pelos profissionais de saúde, de forma a possibilitar o aumento da visibilidade do fenômeno da violência nos serviços de atenção à saúde.

Configura-se, portanto, a necessidade de rastreamento rotineiro da VPI durante o atendimento pré-natal, além da qualificação da atenção por parte dos profissionais podendo resultar na identificação dos casos, assim como no desenvolvimento de tecnologias assistenciais mais aprimoradas(23).

Neste sentido Moran ${ }^{(25)}$ destaca que os enfermeiros apresentam habilidade em estabelecer uma relação de confiança com as pacientes, facilitando o processo de identificação da VPI.

Parte-se do princípio da revelação de um problema de ordem pessoal, para a qual a equipe profissional deve estar atenta, preferencialmente do ponto de vista da abordagem e do reforço da escuta. Por outro lado, acredita-se que a identificação de casos influencie a capacidade de resposta dos serviços de saúde às necessidades das mulheres em situação de VPI, de forma a permitir o estabelecimento de uma linha de cuidados para com as gestantes.

$\mathrm{Na}$ medida em que tal abordagem se inscreve no campo da saúde sexual e reprodutiva, faz-se necessário transpor uma série de valores individuais relacionados às normas culturais e morais, nas quais os profissionais estão submetidos desde a infância. Além disto, a formação profissional, geralmente é caracterizada por modelos formais e conservadores de ensino, que além de transformar os corpos femininos em objetos de estudo e intervenção, consolidam hierarquias de poder e submissão entre as diferentes categorias profissionais, a partir de funções exercidas, particularmente por médicos e enfermeiros ${ }^{(26)}$.

Neste sentido, a violência contra a mulher assume diversos significados de acordo com distintas perspectivas teóricas e disciplinares desenvolvidas, estando condicionados inclusive, às diferenças culturais específicas de cada sociedade.

Minayo $^{(3)}$ sugere que os profissionais da saúde devem somar colaborações interinstitucionais, intersetoriais e da sociedade civil na abordagem dos diversos tipos de violência (educação, serviços sociais, justiça, segurança pública, Ministério Público, Poder Legislativo e movimentos sociais). Propõe também uma ação interdisciplinar entre as ciências sociais, a epidemiologia e a psicologia, que articule teoria, método e estratégias de ações, dada à especificidade do objeto "violência".

Assim, é importante salientar que a problemática da violência não é de responsabilidade única do setor saúde. Este surge como um parceiro complementar à rede intersetorial de apoio à mulheres em situação de violência(3). Entretanto, cabe aos profissionais de saúde, particularmente aos enfermeiros, a detecção de casos de VPI na gestação, de forma a garantir a melhoria da qualidade da atenção e maior efetividade das ações.

\section{CONSIDERAÇÕES FINAIS}

Os resultados sugerem que a VPI está relacionada a condições desfavoráveis de saúde materna, que devem ser criteriosamente investigadas. Na medida em que as queixas das usuárias sejam adequadamente compreendidas e manejadas, efeitos danosos da violência podem ser prevenidos ou minimizados e a rede de apoio pode ser acionada com maior eficiência.

Contudo, as desigualdades de gênero representem o cerne da possibilidade de rompimento do ciclo da VPI, a partir do fortalecimento das mulheres enquanto sujeitos sociais, tanto no âmbito das relações familiares e conjugais, quanto na sociedade como um todo, incluindo-se a importância do seu protagonismo no processo de cuidado à saúde, particularmente na atenção à gestação, ao parto e nascimento.

Acredita-se na contribuição deste estudo ao sensibilizar enfermeiros e demais profissionais de saúde, no que diz respeito à identificação, acolhimento e seguimento das mulheres vitimadas entre os serviços de apoio.

Sugere-se, portanto, que a interface entre saúde sexual e reprodutiva, saúde materna e violência de gênero, seja abordada pela agenda de pesquisas de enfermagem, dada a magnitude e a transcendência dos agravos decorrentes da vulnerabilidade das mulheres, no campo das relações de parceria intima e afetiva, favorecendo o rastreamento e manejo da VPI, assim como os problemas que dela decorrem. 


\section{REFERÊNCIAS}

1. Ellsberg M. Violence against women and the Millennium Development Goals: facilitating women's access to support. Int J Gynecol Obstet. Int J Gynaecol Obstet [Internet]. 2006 [cited 2012 dec 31];94(3):325-32. Availble from:

http://dx.doi.org/10.1016/i.ijgo.2006.04.021.

2. Ângulo-Tuesta AJ. Violência no âmbito Doméstico: a Perspectiva dos Profissionais de Saúde [dissertation]. Rio de Janeiro: Escola Nacional de Saúde Pública; 1997.

3. Minayo MCS, Souza ER. Violência e saúde como um campo interdisciplinar e de ação coletiva. Hist Cienc Saude Manguinhos [Internet]. 1997 [cited $2012 \mathrm{dec} 31$ ];4(3):503-31. Availble from: http://dx.doi.org/10.1590/S0104-59701997000300006.

4. Stetler CB. Updating the Stetler Model of research utilization to

facilitate evidence-based practice. Nurs Outlook [Internet]. 2001

[cited $2012 \mathrm{dec} 31$ ];49(6):272-9. Availble from:

http://dx.doi.org/10.1067/mno.2001.120517.

5. Sanchez SE, Qiu C, Perales MT, Lam N, Garcia P, Williams MA. Intimate partner violence (IPV) and preeclampsia among Peruvian women. Eur J Obstet Gynecol Reprod Biol [Internet]. 2008 [cited 2012 dec 31];137(1):50-5. Availble from: http://dx.doi.org/10.1016/j.ejogrb.2007.05.013.

6. Kim H, Cain R, Viner-Brown S. Intimate partner violence before or during pregnancy in Rhode Island. Med Health R I [Internet]. 2010 [cited 2012 dec 31];93(1):29-31. Available from: http://www.rimed.org/medhealthri/2010-01/2010-01-29.pdf.

7. Johri M, Morales RE, Boivin JF, Samayoa BE, Hoch JS, Grazioso CF et al. Increased risk of miscarriage among women experiencing physical or sexual intimate partner violence during pregnancy in Guatemala City, Guatemala: cross-sectional study. BMC Pregnancy Childbirth [Internet]. 2011 [cited 2012 dec 31];11:49. Available from: http://dx.doi.org/10.1186/1471-2393-11-49.

8. Coonrod DV, Bay RC, Mills TE, Gamble SL. Asymptomatic bacteriuria and intimate partner violence in pregnant women. Am J Obstet Gynecol [Internet]. 2007 [cited 2012 dec 31];196(6):581.e1-4. Available from: http://dx.doi.org/10.1016/j.ajog.2007.03.018.

9. Chu SY, Goodwin MM, D'Angelo DV. Physical violence against U.S. women around the time of pregnancy, 2004-2007. Am J Prev Med [Internet]. 2010 [cited $2012 \mathrm{dec} 31$ ];38(3):317-22. Available from: http://dx.doi.org/10.1016/j.amepre.2009.11.013.

10. Perales MT, Cripe SM, Lam N, Sanchez SE, Sanchez E, Williams MA. Prevalence, types, and pattern of intimate partner violence among pregnant women in Lima, Peru. Violence Against Women [Internet]. 2009 [cited $2012 \mathrm{dec} 31$ ];15(2):224-50. Available from: http://dx.doi.org/10.1177/1077801208329387.

11. Chan KL, Brownridge DA, Tiwari A, Fong DY, Leung WC, Ho PC. Associating Pregnancy With Partner Violence Against Chinese Women. J Interpers Violence. 2011;26(7):1478-1500.

12. Bailey BA, Daugherty RA. Intimate partner violence during pregnancy: incidence and associated health behaviors in a rural population. Matern Child Health J [Internet]. 2007 [cited 2012 dec 31];11(5):495-503. Available from:

http://dx.doi.org/10.1007/s10995-007-0191-6.

13. Audi CA, Segall-Correa AM, Santiago SM, Andrade MG, PerezEscamila R. Violence against pregnant women: prevalence and associated factors. Rev Saude Publica [Internet]. 2008 [cited 2012 dec 31];42(5):877-85. Available from:

http://dx.doi.org/10.1590/S0034-89102008005000041.

14. Durang JG, Schraiber LB. Violência na gestação entre usuárias de serviços públicos de saúde da Grande São Paulo: prevalência e fatores associados. Rev. bras. epidemiol. [Internet]. 2007 [cited 2012 dec 31];10(3):310-22. Available from:

http://dx.doi.org/10.1590/S1415-790X2007000300003.

15. Mendoza Flores ME, Martínez Hernández G, Pizano Zárate ML, Lartigue Becerra T. Violencia de género, embarazo y autoestima en un área urbana de la ciudad de México. Rev. enferm. herediana [Internet]. 2008 [cited $2012 \mathrm{dec} 31$ ]; 1(1):40-7. Available from: http://www.upch.edu.pe/faenf/revfae/Violencia de genero.pdf 16. Brownridge DA, Taillieu TL, Tyler KA, Tiwari A, Chan KL, Santos SC. Pregnancy and Intimate Partner Violence: Risk Factors, Severity, and Health Effects. Violence Against Women [Internet]. 2011 [cited 2012 dec 31];17(7):858-81. Available from:

http://dx.doi.org/10.1177/1077801211412547.
17. Koski AD, Stephenson R, Koenig MR. Physical violence by partner during pregnancy and use of prenatal care in rural India. J Health Popul Nutr [Internet]. 2011 [cited 2012 dec 31];29(3):245-54. Available from:

http://www.ncbi.nlm.nih.gov/pmc/articles/PMC3131125/. 18. Leone JM, Lane SD, Koumans EH, Demott K, Wojtowycz MA, Jensen $J$ et al. Effects of Intimate Partner Violence on Pregnancy Trauma and Placental Abruption. J. Womens Health (Liebert) [Internet]. 2010 [cited $2012 \mathrm{dec} 31$ ];19(8):1501-9. Available from: http://dx.doi.org/10.1089/jwh.2009.1716.

19. Beydoun HA, Tamim H, Lincoln AM, Dooley SD, Beydoun MA. Association of physical violence by an intimate partner around the time of pregnancy with inadequate gestational weight gain. Soc Sci Med [Internet]. 2011 [cited 2012 dec 31];72(6):867-73. Available from: http://dx.doi.org/10.1016/j.socscimed.2011.01.006. 20. Valladares E, Peña R, Ellsberg M, Persson LA, Högberg U. Neuroendocrine response to violence during pregnancy--impact on duration of pregnancy and fetal growth. Acta Obstet Gynecol Scand [Internet]. 2009 [cited $2012 \mathrm{dec} 31$ ];88(7):818-23. Available from: http://dx.doi.org/10.1080/00016340903015321.

21. Beydoun HA, Al-Sahab B, Beydoun MA, Tamim H. Intimate partner violence as a risk factor for postpartum depression among Canadian women in the Maternity Experience Survey. Ann Epidemiol [Internet]. 2010 [cited $2012 \mathrm{dec} 31$ ];20(8):575-83. Available from: http://dx.doi.org/10.1016/j.annepidem.2010.05.011.

22. Silva $E P$, Ludermir $A B$, Araújo TVB, Valongueiro SA. Freqüência e padrão da violência por parceiro íntimo antes, durante e depois da gravidez. Rev Saude Publica [Internet]. 2011 [cited $2012 \mathrm{dec}$ 31];45(6):1044-53. Available from: http://dx.doi.org/10.1590/S003489102011005000074.

23. Salazar M, Valladares E, Ohman A, Högberg U. Ending intimate partner violence after pregnancy: findings from a community-based longitudinal study in Nicaragua. BMC Public Health [Internet]. 2009 [cited $2012 \mathrm{dec} 31$ ];9:350. Available from:

http://dx.doi.org/10.1186/1471-2458-9-350.

24. Schreiner MT. O Abuso sexual numa perspectiva de gênero: o processo de responsabilização da vítima. Fazendo Gênero 8: Corpo, Violência e Poder. Florianópolis, 2008.

25. Moran BA. Intimate partner violence: the difference nurses can make. Nurs Womens Health [Internet]. 2008 [cited $2012 \mathrm{dec}$ 31];12(3):194-6. Available from: http://dx.doi.org/10.1111/j.1751486X.2008.00320.x.

26. d'Oliveira AF, Diniz SG, Schraiber LB. Violence against women in health-care institutions: an emerging problem. Lancet [Internet]. 2002 [cited 2012 dec 31];359(9318):1681-5. Available from: http://dx.doi.org/10.1016/S0140-6736(08)61345-8.

Artigo recebido em 21/10/2011.

Aprovado para publicação em 20/03/2012.

Artigo publicado em 31/12/2012. 